a rearrangement of the atoms resulting in a less complex molecule. We also know that organic cells are made up of rather unstable molecules. I can not but think that we have here to do with such a change. The radiant energy of the $X$-rays which are of comparatively long wave length, would be absorbed by the molecules of the first substance coinciding with their absorption band encountered, and if these molecules were unstable, they would suffer rearrangement of their atomic structure. Such a rearrangement, it is more than likely, would be, if carried far, sufficient to greatly modify the cell function and produce cell death. Apparently this is the exact condition produced, for the dermatitis shows every indication of being a superficial gangrene, and the sloughing process continues until the dead material has been cast out, when healing is spontaneous.

I have observed that dermatitis is more apt to ensue from exposure to low vacuum tubes than to tubes of higher vacuum, and that a very long exposure may be safely made, when the tube is of excessively high vacuum and the rays emitted are of great penetration. In the former case the vast majority of rays are unquestionably absorbed by the skin, whereas in the latter case but few rays are absorbed.

We should also expect, if this theory is true, to see more or less disturbance of the subcutaneous tissues following excessive exposures; and such is the case, for we find that prolonged exposure has resulted in sloughing of the true skin and underlying tissue; and that pain and soreness in and of the bones and joints has been observed repeatedly; and that cases of miscarriage in the latter months of pregnancy have followed long exposure to these rays. The evidence seems to me conclusive.

The possible danger of dermatitis or other inflammatory process, should not deter us from employing this diagnostic aid. In the vast majority of cases no untoward consequence need be anticipated, if a few precautions are borne in mind. The tube should be carefully examined and not used for diagnostic purposes when the vacuum is low; the minimum exposure necessary to secure a satisfactory skiagraph, when the tube is not too low, and the distance of the tube from the surface of the part exceeds six inches, will almost never produce a dermatitis. When the case is one requiring a prolonged exposure, or several repeated exposures, it becomes a matter of judgment, whether the urgency of the examination is sufficient to warrant the dermatitis which may ensue.

803 Sutter Street.

\section{THE FALLACIES OF X-RAY PICTURES.} BY EDWARD A. TRACY, M.D.

FELLOW OF THE MASSACHUSETTS MEDICAL SOCIETY; MEMBER OF THE AMERICAN MEDICAL SOCIETY. BOSTON.

Roentgen's marvelous work on the properties of the X-rays (their nature is as yet unknown) has already been productive of much good in surgery and medicine-almost entirely in the field of diagnosis. The application of his discovery necessitates the rewriting of the text-books on fractures and dislocations. Facts, heretofore "smothered in surmise" are clearly set forth by the radiographs. For example, no writer, of which I am aware, on fractures, suspected the frequency with which fracture of the ulnar styloid process accompanies Colles' fracture; yet this frequency has been demonstrated by radiographs of Colles' fractures.
While much has been gained in accuracy of diagnosis by the aid of $\mathrm{X}$-ray pictures, there is one branch of practical medicine where harm is threatened by their employment. I refer to medical jurisprudence. X-ray pictures have been already admitted as evidence in some courts. Their indiscriminate admission will hurt the cause of justice-because they can easily lead to fallacy or error. Their use as evidence of injury, is only safe when certain conditions have been ful-

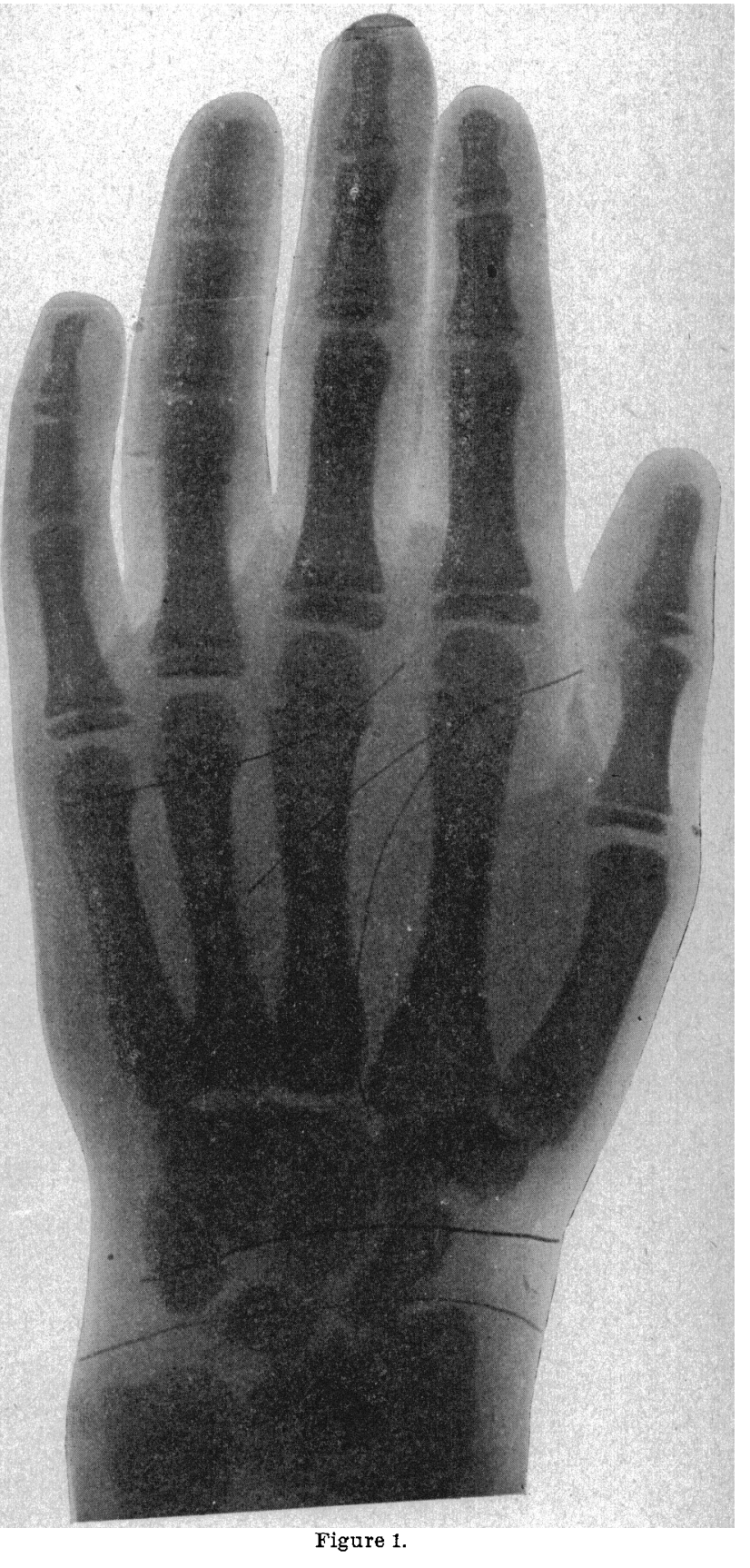

filled, in their taking and presentation. I shall briefly indicate further on what these conditions are.

In all $\mathrm{X}$-ray pictures there is distortion. The reason is X-rays emanate from a point, and are not parallel. Interference with these rays follow the ordinary physical and mathematical laws of rays emanating from a point. (Of course it is understood that there is neither reflection nor refraction of the X-ray.) Thus the nearer to their source is an obstruction to the rays, the larger will be the resultant shadow or picture; the 
size of the shadow depends also upon the nearness of the object to the surface upon which the shadow falls; the further the surface from the object, the larger the shadow. If we had for a source of $X$-rays a surface as large as the object to be pictured, there would be no distortion, for the $\mathrm{X}$-rays would be parallel. X-ray pictures in that case would be easy of comprehension, and never misleading. To read correctly the lesson of an X-ray picture, the obliqueness of X-rays must be kept in mind, and mental correction made for the disproportion and distortion caused by this obliqueness.

To illustrate the foregoing remarks, I present Figs. 1 and 2 . They are plates of radiographs of the same hand, the right, of a boy 13 years of age. Before tak-

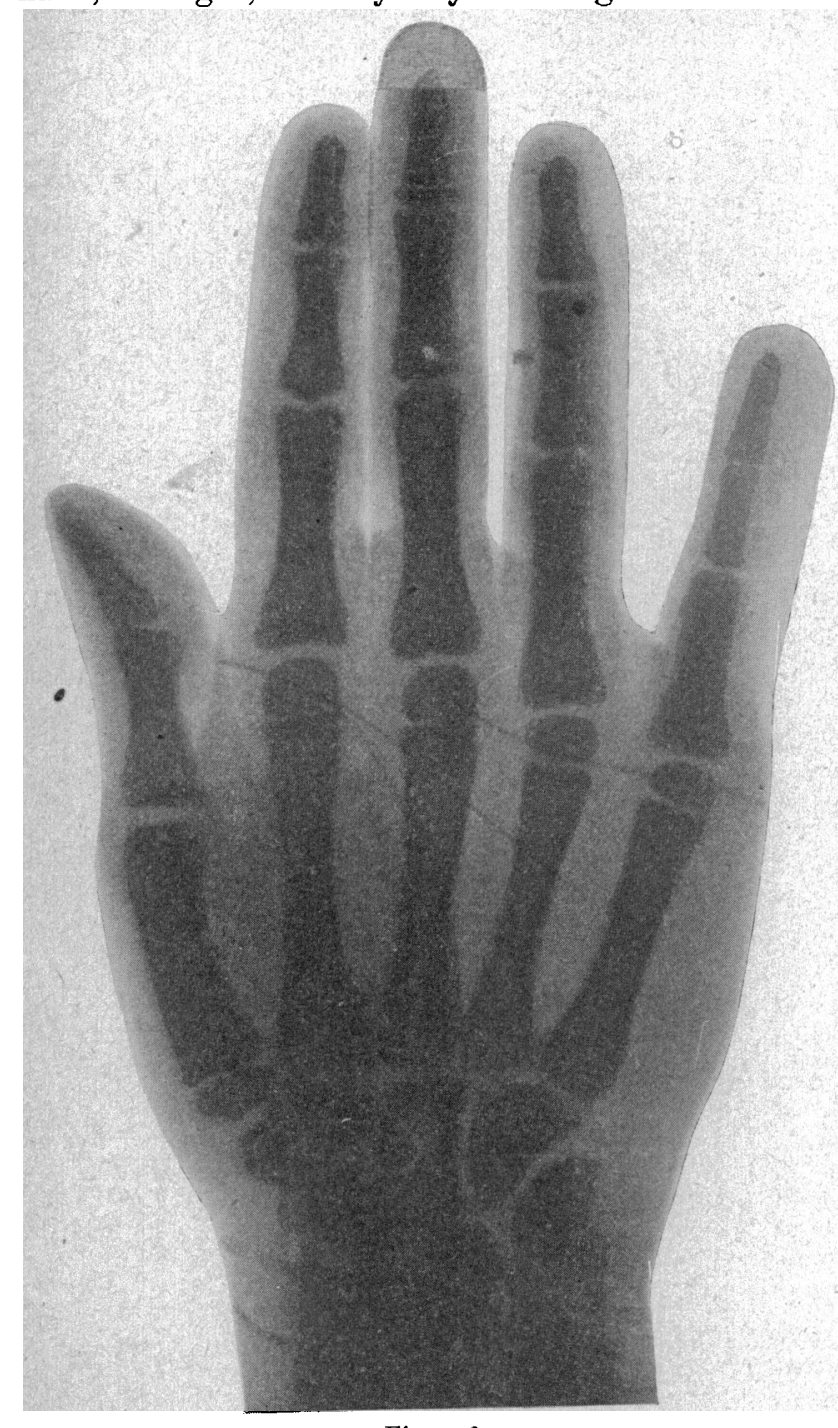

Figure 2.

ing them, I thought that the relations of the palm and wrist lines to the underlying bones could be accurately determined by radiography. My plan was this: I covered the palm and wrist lines with pieces of copper wire. The wire was attached to the skin by means of collodion and cotton fiber. The copper wire being resistant to penetration by the $\mathrm{X}$-rays would cause dark lines upon the picture, and thus I hoped to have the palm and wrist lines represented with the bones, and their relations shown. The palm and wrist lines are represented but their relations are not accurately pictured. This is proven by the plates. Fig. 1 is from a radiograph of a hand with its palm toward the sensitized plate. Fig. 2 is of the same hand turned over so that its back was toward the plate. The relation of the palm and wrist lines to the bones had not actually changed by simply turning over the hand; the pictures represent these relations to have changed, and therefore are fallacious. If our source of $X$-rays was so large that the rays streaming through the object were parallel, such divergence would not exist, and the lines representing those of the palm and the wrist would coincide in both pictures.

Besides the want of coincidence in the palm and wrist lines in both pictures, I invite attention to the difference in size of the thumb metacarpal in the pictures. In Fig. 2 the thumb metacarpal is pictured as thicker than in Fig. 1. The pictures represent a difference in size where none exists, as both are pictures of the same bone. Hence, again, $\mathrm{X}$-ray pictures are fallacious. This difference in size is explained thus: In Fig. 1 the hand was palm against the sensitized

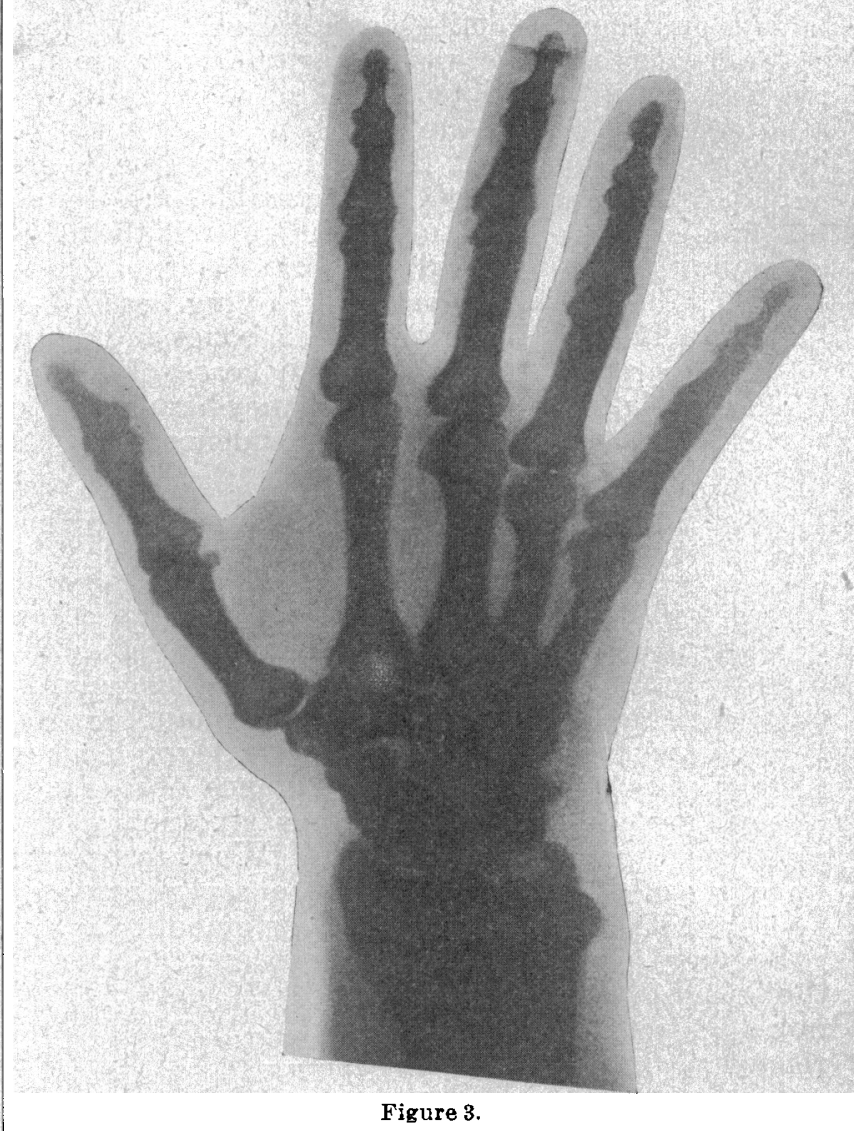

surface of the plate, and therefore the thumb metacarpal bone was nearer to the plate, than when the hand was with its back against the plate as in Fig. 2 . The thumb being nearer the plate in the first position than in the second, its shadow was smaller, so pictured in Fig. 1.

Besides the variations in the picture caused by the distance of the object from the source of the X-rays, and the variations caused by the distance of the object radiographed from the sensitized plate, there are variations caused by the position of the object with relation to the direction in which the $\mathrm{X}$-rays strike the object. This is shown by Figs. 3 and 4 . In Fig. 3 we have the bones shown thicker than in Fig. 4 and with curious spur-like exostotic deposits upon the metacarpal bones. Both are radiographs of the same 
hand. The difference in the pictures was caused by the difference in position of the Crooke's tube when they were taken. In taking picture 3 the tube was more to the ulnar side of the hand than when taking picture 4.

In my possession is a plate in which the deformity caused by Colles' fracture is quite closely simulated by a similar change in the position of Crooke's tube. The plate is of a normal wrist. The importance of this from a medico-legal point of view need not be enlarged upon.

I have thus shown X-ray pictures to be fallacious and misleading-and described some of the causes of these fallacies.

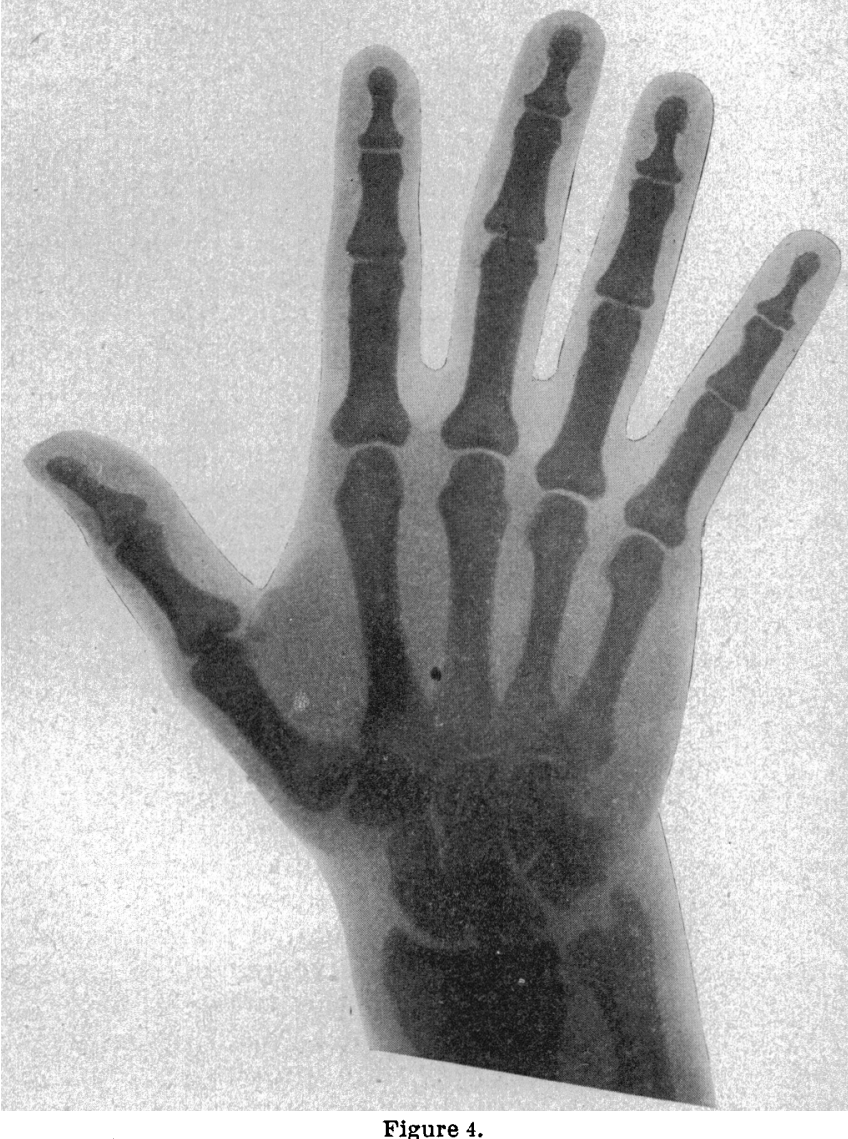

Because X-ray pictures can be fallacious, should they be excluded from court as evidence? Certainly not. It is well to know their limitations, and to remember that appearances may deceive. $X$-rays, properly used, are as a search-light in the exposition of bone lesions. But the lesions must be pictured from different directions, and the resultant pictures compared with pictures of the normal opposite member. Moreover the pictures of the injured member and those of the opposite normal member, must be taken with the same relative positions of the Crook's tube, the limb and the sensitized plate. Then can truth be arrived at, and truth is essential for justice.

99 Broadway.

Salicylate of Methyl in Scarlet Fever Arthralgia.-Professor Roger has secured rapid and marked improvement in the arthralgia accompanying infective diseases and especially scarlet fever, by the use of salicylate of methyl. Twenty to forty crops are applied to the articulation morning and night, which is afterward wrapped in oiled silk.-Semaine Méd., A ugust 18.
CORNEAL BURNS FROM ACCIDENTS IN THE USE OF CURLING IRONS. REPORT OF SIX CASES.

JOHN O. McREYNOLDS, B.Sc., M.D.

DALLAS, TEXAS.

This unusual form of traumatism is worthy of mention, because of the severity of the pain, the grave apprehensions of the patient and the uniformly speedy and complete recovery. In the six cases that have recently come under my observation (Mrs. W., Miss L., Miss E., Miss M., Miss L. and Miss H.) the injury was received directly upon the cornea by dropping the hot curling-iron upon the open upturned eye. The extent of the injury has varied from three square millimeters in area to a third of the corneal surface.

In the severer cases the pain has been very intense unless continually relieved by local anesthetics. The seared superficial epithelium in a few hours exfoliates in a white opaque mass, leaving beneath a denuded corneal surface, which soon becomes, under favorable treatment, re-covered with normal epithelium and within a few days the eye is perfectly well. In corneal injuries in general I should object to the employment of cocain, because of its bad influence upon nutrition, but in this form of injury, in which the pain is so excruciating and the danger of subse. quent ulceration so slight, I have considered it prudent to use a weak solution or ointment of cocain or eucain to secure rest for the patient. For this particular purpose the eucain has the advantage over cocain in that it increases rather than diminishes corneal nutrition and also is capable of being thoroughly sterilized by heat. The additional indications are the frequent use of mild antiseptic solutions, the moderate employment of a mydriatic and the application of a light bandage to obtain rest and protection for the wounded eye.

\section{GENERAL IMPRESSIONS FROM SIX YEARS} USE OF THE OLD TUBERCULIN.

Read before the British Medical Association at Montrea], Sept. 2, 1897. BY JAMES T. WHITTAKER, M.D.

Robert Koch announced the discovery of tuberculin at the Tenth International Congress at Berlin, August, 1890.

The publicity of the occasion, the fame of the author, above all the defiance of the disease to all treatment hitherto, lent peculiar interest to the dis. covery. The suddenness of the announcement was another factor. No one had even an inkling of the work leading up to it.

The declaration of the discovery of the tubercle bacillus in 1882, though credited by many of the members of the critical audience in the Berlin Physiological Society to which it was presented, met with so much scepticism in the profession at large as to have been practically disbelieved. Most of the leading medical journals discredited it; many of them derided it. When the truth asserted itself the disbelievers found themselves punished for incredulity. So, although the problem was harder. belief was easier on this occasion. In fact, there was reaction to the other extreme and more was expected of the remedy than was expressed in the modest claims of the author. This was the reason why medical men, who represent the most cautious and conservative branch of human culture, accepted the remedy at once 